\title{
The Effect of Past and Future Economic Fundamentals on Spending and Pricing Behavior in the FRB/US Macroeconomic Model
}

\author{
Peter von zur Muehlen*
}

February 16, 2001

\begin{abstract}
This paper derives and presents mean leads and lags as well as patterns of relative importance weights implied by the PAC (polynomial-adjustment-cost) error-correction equations which form the core of the FRB/US model at the Federal Reserve Board. Relative importance weights measure the contributions of past and future expected changes in fundamentals on current decisions. These and the associated mean lags and leads can be considered summary measures of key dynamic properties of FRB/US. The spending equations are those for total consumption, durable consumption, business equipment, residential housing, and private inventories. The pricing equations are those for the price level and wage growth. In addition FRB/US has one PAC equation for dividends and one for labor hours.
\end{abstract}

\section{JEL Classification: C3,C5,E1}

Keywords: Macro modeling, dynamics, expectations, polynomial adjustment costs, error correction, mean lags and leads.

${ }^{*}$ Board of Governors of the Federal Reserve System. I would like to thank David Reifschneider for his helpful comments. 


\section{Introduction}

Two principles guide the specification of behavioral equations in the FRB/US macroeconomic model of the U.S. One is the assumption of optimizing behavior by rational participants in the economy who seek to achieve highest levels of welfare and profits. The other is the presence of frictions that (except in the case of pure asset prices) prevent instantaneous achievement of goals set by optimizing agents. ${ }^{1}$ A corollary of these two principles is that in their decisions, firms and households look forward and make plans for the future, giving expectations a pivotal role in modeling the US economy.

To put these principles into effect for modeling the dynamic behavior of the US economy, FRB/US introduces the distinctive feature of explicitly separating expectations of future events from any delayed responses to them. Expectations are, of course, by nature unobservable, and so they need to be modeled. FRB/US generates estimates of expectations held by individuals and firms using the forecasts of two alternative representations of the economy: (1) either a small VAR model, auxiliary to the large model and assumed to be known to all agents, or (2) FRB/US itself. In the latter case, households and firms are assumed to have a detailed understanding of the entire economy, as represented by the model. To the extent that FRB/US is a true representation of the economy, such "model consistent" expectations are considered "rational." Underlying the alternative VAR approach to expectations formation is the assumption that knowledge is more limited or segmented and that agents economize on information by focusing on historical interactions among three macroeconomic variables: the rate of inflation of consumption goods; the federal funds rate, reflecting monetary policy; and the gap between real output and its trend level, reflecting the cyclical state of the economy. ${ }^{2}$

As noted above, the need for forming expectations in decision making other than asset pricing arises from delays in carrying out plans that are delayed by frictions and constraints

\footnotetext{
${ }^{1}$ The FRB/US macroeconomic model is documented in Brayton and Tinsley ((eds.), 1996), Brayton, Levin, Tryon and Williams (1997b), and Brayton, Mauskopf, Reifschneider and Williams (1997a)

${ }^{2}$ In principle, the VAR approach to modeling expectations need not be "irrational." If decision makers engage in learning and revise their parsimonous "rest-of-the-world" VAR model in accordance with new information, the interplay between decisions based on forecasts from that model and its evolution implies eventual convergence toward expectation that are consistent with the large and presumably true model of the economy.
} 
in dynamic adjustments, which may differ across sectors. The presence of frictions in adjustment imposes a necessity to look ahead: the longer it takes to reach a desired (or targeted) level of expenditure, the longer will be the necessary forecast horizon; and if adjustment is very slow, decisions made today will be more heavily influenced by expectations farther out in the future, meaning that greater weight will be given to expected events in the distant future.

Frictions arise from a variety of sources. One is incompleteness of information on which to base decisions. If acquiring information is costly, efforts to learn about the environment, using, for example, signal extraction, search, and experimentation methods, will lead to a protraction of initially formed plans. Another source, affecting scheduling and delivery, is purely mechanical and depends on the economy's communications, transportation, and production infrastructure. Institutional arrangements, such as price and wage contracting over multiple periods also serve as impediments to instantaneous action. For example, Calvo (1983) showed that if expiration dates of wage contracts are randomly distributed over the economy, the aggregate of wages in any period is a weighted average of unexpired contract wages. Finally, as first proposed by Eisner and Strotz (1963), capital, labor, and other business expenditures are subject to convex costs of adjustment, costs that show up as reductions in profit.

Instances of frictions are familiar to economists. In the case of capital investment, the time-to-build argument is familiar: it takes time to procure and install equipment and to train workers to use it because often, many steps and negotiations come between initial conceptualizations, drawing of plans, developments of complete specifications, and final delivery of a satisfactorily engineered machine. In many instances, it is more expensive to get accelerated delivery from suppliers. In the case of residential housing expenditures, the time between a change in income or the mortgage rate and expenditure on a residence is filled with planning, search, construction, loan applications, etc.-all things that delay ultimate settlement. Adjustments in hourly compensation may be slowed by a number of factors, including such contractual arrangements as alluded to above, delays in workers' recognition of a change in the real wage, fear of effects on morale in the case of downward adjustments, and many similar factors familiar to labor economists. Even prices are sluggish. Under the assumption of markup pricing over unit costs, delays in the adjustment of 
wages can affect the pattern of price adjustments over time. However, firms can and do excercise considerable discretion over markups, necessitated for example by instantaneous fluctuations in the prices of intermediate commodities used as inputs.

Because of the variety of sources of delays, the paradigm for friction in FRB/US is a generalization of the standard theory of convex adjustment costs; this generalization was developed by Tinsley (1993). As is well known, standard econometric models that impose quadratic costs of adjustment have not done well in representing the dynamic behavior of macroeconomic variables. Whereas quadratic adjustment costs are by definition confined to first-order differencing effects, the generalized model, named PAC for "polynomial adjustment costs," permits richer dynamics within a theoretically-based framework that remains parsimonious and is amenable to testable restrictions. The PAC model comprises a generalized class of specifications for frictions in economic activity, including not only costs of $\mathrm{k}$-th-order differencing but also costs associated with changes in moving averages, such as might be associated with seasonal or term contracts and time-to-build capital expenditures.

The implied Euler conditions lead to decision rules that can be represented as rational error-correction processes. ${ }^{3}$ These differ from conventional error-correction models in two important ways. First, higher-order autoregressive lags of the decision variable will now appear in the error-correction model, where the degree of the autoregression is one less than the polynomial degree specified in the adjustment cost criterion. The PAC formulation therefore offers a plausible theoretical justification for high-order autoregressivity often found in empirical error-correction models of important macroeconomic variables in the US economy. Second, the term capturing forecasts of future changes in the planned or targeted activity of the modeled variable now becomes subject to more complex discounting, involving the multiple roots of the higher-order polynomial adjustment cost function. This means that the relative importance of expected changes in the future equilibrium path of a variable to its current behavior hinges on the exact nature of frictions that characterize the behavior of that variable.

This last comment suggests a useful way of viewing the implications of any particular

\footnotetext{
${ }^{3} \mathrm{~A}$ version of the theory of rational error correction, developed by Tinsley (1993), was published by Kozicki and Tinsley (1999).
} 
dynamic specification, one that reveals just how a series responds to its own past and future fundamental-its equilibrium path. The optimal decison rule requires that at any time, an action that is subject to friction will be influenced by partially fulfilled plans for adjustment formulated in earlier periods and to revisions to plans anticipated in future periods, based on current information. As a consequence, decisions and actions in the current period can be expressed as weighted averages of the equilibrium values inherited from past periods and the target path anticipated in the future, where the weights measure the relative contributions of past and future expected fundamentals to current levels of the decision variable. It should come as no surprise that since different economic variables are subject to unique forces and frictions, each series will have its characteristic signature pattern of weights influencing its movements over time. These patterns are derived and discussed in the remainder of this paper.

\section{Adjustment Dynamics in FRB/US}

As described in Brayton and Tinsley ((eds.), 1996), firms and households calculate desired equilibrium paths of expenditures or prices, having formed expectations generated by either of the two methods mentioned above. At time $t$, the equilibrium level of a variable $y_{t}$ is denoted $y_{t}^{*}$. FRB/US adopts a single paradigm to represent dynamic adjustments of major decision variables as follows: Participants seek to balance the expected cost of deviating from the equilibrium path, defined as the infinite discounted sum of the squared differences between $y_{t+j}$ and $y_{t+j}^{*}$, where $j$ ranges from zero (current time) to infinity, against the costs of making changes:

$$
E_{t-1} \sum_{j=0}^{\infty} \beta^{j}\left[\left(y_{t+j}-y_{t+j}^{*}\right)^{2}+b_{1}\left(\Delta_{t+j} y_{t+j}\right)^{2}+b_{2}\left(\Delta^{2} y_{t+j}\right)^{2}+\cdots+b_{m}\left(\Delta^{m} y_{t+j}\right)^{2}\right],
$$

where $E_{t-1}\{$.$\} is a forecast of future costs based on information available at the end of$ the preceding period, $t-1, m$ is the number of $b$ coefficients in the cost function, and $\beta$ is a discount factor between zero and one. The first squared term is the cost incurred if the variable, $y$, deviates from its equilibrium level, $y^{*}$, in period $t+j$. The remaining 
terms measure the expected costs of frictions that arise with changes in $y$. The letter, $\Delta$, denotes change, so that $\Delta y_{t+j}=y_{t+j}-y_{t+j-1}$ is the change in the level of $y$ from period $t+j-1$ to $t+j$, and $b_{1}$ is the unit cost of making this change. In the next term, $\Delta^{2} y_{t+j}=\Delta\left(y_{t+j}-y_{t+j-1}\right)=\left(y_{t+j}-y_{t+j-1}\right)-\left(y_{t+j-1}-y_{t+j-2}\right)$ is the change in the change. In this instance, $b_{2}$ represents the unit cost of accelerating $y$. In the following term, $b_{3}$ is the unit cost of changing the rate of acceleration.

The outcome of this minimization is an adjustment process that acts to gradually eliminate the remaining gap between a price or a spending level and its desired level in a way that depends on future expected changes in the target level as well as on past changes in the decision variable. Formally, the decision rule that minimizes (1) is the following, ${ }^{4}$

$$
\Delta y_{t}=-a_{0}\left(y_{t-1}-y_{t-1}^{*}\right)+\sum_{j=1}^{m-1} a_{j} \Delta y_{t-j}+E_{t-1}\left\{\sum_{i=0}^{\infty} f_{i} \Delta y_{t+i}^{*}\right\}
$$

This is a generalized error correction equation which is influenced by past changes in $y$ and by expected changes in the future equilibrium path, $y^{*}$. The optimal adjustment of $y$ toward its equilibrium is determined by: (1) last period's deviation of $y$ from its equilibrium level, $y_{t-1}-y_{t-1}^{*}$, (2) past changes in the level of $y$, and (3) a weighted forecast of future changes in equilibrium levels, $y^{*}$. The forecast weights, $f_{i}$, are functions of the discount factor, $\beta$, and the unit cost parameters, $b_{1}, b_{2}, \cdots$. It is important to note that lagged changes in $y$ beyond $t-1$ would not appear in (2) were it not for the presence of adjustment cost terms of order higher than quadratic, those involving $b_{2}, b_{3}$ and so on. Indeed, the autoregressive order in (2) is exactly $m-1$. In the estimated PAC equations for durable equipment spending, the price index for adjusted final sales (the principal deflator of the model), and the growth rate of employee compensation, lags exceeding 1 cannot be rejected, thus presenting a plausible case for higher-order adjustment costs in at least these three instances. ${ }^{5}$

The optimal level of activity, $y_{t}$, can be represented as a two-sided moving average of

\footnotetext{
${ }^{4}$ See Tinsley (1993) for details.

${ }^{5}$ The estimated higher-order terms in $\Delta y$ can plausibly mimic more generalized moving average processes, such as would be implied by time-to-build, learning-by-doing, or staggered contracting.
} 
past and future equilibrium values, $y^{*}$,

$$
y_{t}=E_{t-1}\left\{\sum_{j=-\infty}^{\infty} w_{j} y_{t+j}^{*}\right\}
$$

where the weights, $w_{j}$, sum to one. ${ }^{6}$ This equation says that the current level of $y$ is, in part, the legacy of earlier fundamentals, $y^{*}$, and, in part, the anticipation of its future equilibrium path. One advantage of this equation is its parsimonious appearance. A second benefit is that the bell-shaped pattern of the weights plotted in Figures 1 and 2 provide a simple and intuitive view of the dynamic behavior that is characteristic to each activity. One would expect variables subject to significant adjustment costs to be far more influenced by distant events than variables that move more or less unimpeded.

\section{The Distribution of Lead and Lag Weights}

The FRB/US model contains nine PAC equations involving polynomial adjustment costs: spending on nondurable goods and services; spending on consumer durable goods; investment in residential housing, producers' durable equipment, and trade inventories; aggregate labor hours; the price level; and the growth rates of employee compensation and dividends. By contrast, yields on equities and bonds, and the exchange rate are modeled as asset valuation equations. This means that valuations, while forward-looking, are not constrained by adjustment frictions: they are purely forward looking. ${ }^{7}$

Figures 1 and 2, drawn at identical scales to facilitate comparisons, display the relative importance weights for all the PAC equations in FRB/US and of the yield on a ten-year Treasury bond. The area under each curve sums to 2 , since each point on a plot measures the weight, $w_{i}$, relative to its backward or forward sum. So, for example, points to the right of center, depicting forward weights, are the $w_{i}$ divided by the sum of all weights to the right of the center. Similarly, relative-importance lag weights are computed based on their sum going backward. Each point to the right of center therefore shows the contribution of $y^{*}$ to

\footnotetext{
${ }^{6}$ The distribution of weights is derived in Appendix A.3.

${ }^{7}$ Full details about the specification of these equations are given in Brayton and Tinsley ((eds.), 1996).
} 
the current decision as a fraction of the contributions of all future expected $y^{*} .{ }^{8}$ Weights for past quarters, shown to the left of the peaks centered at zero, indicate the relative importance of past equilibrium levels to current decisions. Because, as time passes, older plans become completed, the relative weights for past planning periods approach zero. Conversely, the relative weights of more distant future weights, shown to the right of zero, gradually vanish because of discounting and because more distant future needs can be satisfied by corrective actions in future quarters.

In Figures 1 and 2, we can broadly distinguish two types of weight distributions. One shape, typified by expenditures on consumption, equipment investment, the price level, wage growth, and, most notably, dividends, tends to be relatively flat, indicating a strong influence of planning considerations in the evolution of these variables. In the case of durable equipment expenditures, for example, the shape of the curve conforms to well known priors about the sluggishness of capital goods investment: it is slowed considerably by frictions and adjustment costs, leading to a pattern that gives much weight to both past changes and to expected future planned changes. Although firms, in principle, plan over an infinite horizon, the effective length of the planning period is limited by the extent of frictions associated with a firm's actions. The similarity of the patterns for consumption and equipment expenditures at frequencies in excess of one year is likely a reflection of firms' setting their capital spending plans in anticipation of future sales.

The second group of shapes, typified by inventories, residential housing expenditures, and during the first year, durable consumption, exhibits relatively large weights at nearterm frequencies. Inventory accumulation tends to be a fast reacter compared to wages and prices, with very little adjustment needed after the first three to four quarters. Although firms have, at least theoretically, the ability to adjust wages, hours, prices, and inventories in response to demand shocks, it appears to be typically inventories that bear the initial brunt, with hours following as the next option. ${ }^{9}$ Likewise, housing expenditures react very quickly, indeed, fully within two years. This is consistent with the well known tendency of

\footnotetext{
${ }^{8}$ Under the asssumption that agents in various sectors plan their actions rationally, the required rate of return, including a risk premium, received by investors and households is approximated by the real rate of return to equity.

${ }^{9}$ The emergence of just-in-time inventory management may change this ordering so that manipulation of inventories may become less of an option.
} 
housing expenditures to be among the first to react to changes in monetary policy. The relatively fast movement of housing expenditures is consistent with a slowly changing housing stock characterized by a low depreciation rate. One might note that a certain disparity between stock and flow adjustment also describes other forms of capital. A comparison between the weight patterns of prices and wages shows further that the former have a much shorter effective time horizon than the latter. This apparent lack of dynamic coordination suggests that mark-ups over unit labor costs are flexible, allowing prices to adjust more quickly than wages, which tend to be set contractually over several periods. Inventories and the price level as well as wage growth experience some degree of overshooting behavior within one or two years: in the first two cases, the weight density includes negative values for the influence of equilibrium stocks and prices, respectively, beyond two years from the current date, while wages react negatively to equilibrium wages more than 4 years away.

In Figure 2 the characteristic weight pattern of a purely foward-looking asset price, the ten-year treasury bond yield, has been added for illustrative purposes. The ten-year yield is determined by a term structure equation which forecasts the federal funds rate over the maturity of the bond. Note that the relative importance weights of expected future funds rates decline in a straight line over the ten-year planning period. The associated mean lead is about four years, roughly half its mean duration.

\section{Implied Mean Leads and Lags}

A summary measure of the effective average length of the forward planning period for a variable is the mean lead implied by its relative-importance weights. The mean lag of a series is useful as a measure of its characteristic average speed of response to unexpected shocks, i.e., events, for which planning is, by definition, impossible. Mean leads measure the average responsiveness to future expected events and are a reflection of the typical decision horizon for a variable. The mean lags and leads for the six series discussed here 
are shown in Table $1 .{ }^{10}$ As indicated in the table, inventories, prices, and hours, for which frictions are among the least, compared with those of other series considered here, exhibit very similar mean leads and lags of less than a year, apparently reflecting firms' abilities to adjust these decisions variables quickly and interchangeably as predicted by theory. The shortest lags are those for residential housing expenditures, whose mean lead and lag is $1 / 2$ year. This may be explained by production function features in construction: typically low capital intensity in the residential construction industry allows relatively quicker responses to changes in interest rates and in expected prices and income. Likewise, financing at the residential level is fairly qick and efficient. Durable equipment and consumption expenditures, characterized by typically longer planning horizons, have mean leads of 1-1/2 years, as does wage growth. Interestingly, it is dividend payouts that exhibit the greatest persistence. Although earnings tend to be volatile, firms have incentives to smooth dividend payouts to avoid giving their stock holders false signals of fundamental events that are likely not as variable. Investors who own equity for their income stream may be discouraged from owning a stock if its dividends are unreliable; for the same reason, firms may be disinclined to raise dividends unless they are sure that earnings will remain high in the future. This suggests that dividend payouts can be interpreted as signals of the underlying fundamentals that drive cyclical earnings. Another explanation for highly persistent dividend patterns may be firms' desire to maintain maximum flexibility with retained earnings to finance investment.

For prices, the mean lag is four quarters, the same as its mean lead. The mean response lag for durable equipment expenditures is roughly one and a half years.

\footnotetext{
${ }^{10}$ An exact method of calculating mean leads and lags is presented in the appendix. For the truncated horizon, $T \leq \infty$, an approximation of the mean lead of a series is the product of the sequential number of each quarter in the forward planning period and the corresponding relative-importance weight,

$$
\bar{w}=\sum_{i=0}^{T} i \frac{w_{i}}{\sum_{i=0}^{T} w_{i}},
$$

where $\frac{w_{i}}{\sum_{i=0}^{T} w_{i}}$ is the relative-importance weight for the i-th quarter in the planning period. Mean lags are calculated similarly.
} 
Table 1: Equation Coefficients and Implied Mean Leads and Lags

\begin{tabular}{lllll|cc}
\hline \hline & \multicolumn{7}{c|}{$t-1$} & $t-2$ & $t-3$ & & \\
Equation & $-a_{0}$ & $a_{1}$ & $a_{2}$ & $a_{3}$ & Mean Lag & Mean Lead \\
\hline Durable Equipment & .095 & .092 & .232 & & 6.16 & 5.56 \\
Inventories & .110 & .544 & & & 3.14 & 3.07 \\
Consumption & .119 & .081 & & & 6.70 & 5.81 \\
Durable Consumption & .197 & -.147 & & & 4.80 & 4.30 \\
Housing Expenditures & .155 & .478 & & & 2.35 & 2.32 \\
Price Deflator & .082 & .339 & .258 & & 3.95 & 3.95 \\
Wage Growth & .058 & .192 & .237 & .184 & 5.70 & 5.70 \\
Hours & .124 & .402 & & & 3.83 & 3.60 \\
Dividends & .043 & .399 & & & 13.0 & 10.4 \\
\hline \hline
\end{tabular}

\section{Implied Polynomial Cost Parameters}

As shown in Appendix A.2, it is possible to retrieve the implied polynomial adjustment cost parameters from the estimated error-correction equations. These are shown in Table 2 . The presence of second and third lags in only durable equipment expenditures, wage growth, and the price deflator suggests that higher-order frictions are not common in the U.S. economy. Observe that a high order in a variable's polynomial adjustment cost function does not necessesarily imply a high mean lead or lag of its series, since these are determined by the implied eigenvalues of the polynomial, shown in Table 3.

\section{Two Caveats}

For each decision variable, the estimated lead and lag patterns in Figures 1and 2 are based on a single-equation, unitary error-correction model, estimated independently of other decision variables that might influence its behavior. ${ }^{11}$

\footnotetext{
${ }^{11}$ Some preliminary empirical findings by von zur Muehlen (1993) suggest that for a general equilibrium vector rational error-correction model of multiple factor demands by a firm that maintains both final and intermediate inventories, the movements in factor inputs and inventories are influenced by disequilibrium gaps of all other decision variables under the control of the firm in varying degrees.
} 
Table 2: Polynomial Cost Parameters Implied by Estimates

\begin{tabular}{lrrrr} 
Equation & $b_{1}$ & $b_{2}$ & $b_{3}$ & $b_{4}$ \\
\hline Durable Equipment & 0.30 & 1.01 & -0.23 & \\
Inventories & 0.04 & 0.54 & & \\
Consumption & 0.72 & 0.08 & & \\
Durable Consumption & 1.14 & -0.15 & & \\
Housing & 0.05 & 0.48 & & \\
Price Deflator & 0.00 & 1.29 & -0.26 & \\
Wage Growth & 88.7 & -19.8 & -1.30 & 0.18 \\
Hours & 0.19 & 0.40 & & \\
Dividends & 0.31 & 0.40 & &
\end{tabular}

The foregoing discussion also abstracts from the likely presence of asymmetries in dynamic responses to shocks with respect to sign and phase of business cycle if frictions and/or adjustment costs for any of the variables treated here asymmetric with respect to business cycle phases. For example, it is easier to raise prices, even in the face a potential loss of customer base, than to replenish stocks when economy-wide capacity is strained. Similarly, downward nominal wage adjustment is less constrained when unemployment is high than it would be under normal conditions. The individual estimated error-correction equations on which the weight patterns are based, represent average responses over business cycles and thus do not reflect asymmetry, per se. ${ }^{12}$

\footnotetext{
${ }^{12}$ Tinsley and Krieger (1997) examine asymmetries in price and output adjustments in SIC two-digit industries and find that asymmetric pricing appears to be structural, with pricing decisions depending on the position of the industry price in relation to its trend.
} 


\section{Table 3: Eigenvalues of Dynamic Equations}

\begin{tabular}{lllll}
\hline \hline & \multicolumn{5}{c}{ Eigenvalues } \\
\hline Durablion & \multicolumn{5}{c}{ Equipment } & .83 & .62 & -.45 & \\
Inventories & .74 & .74 & & \\
\hline \hline Consumption & .87 & .09 & & \\
Durable Consumption & .83 & -.18 & & \\
Housing & .69 & .69 & & \\
Price Deflator & .83 & .83 & -.37 & \\
Wage Growth & .88 & .88 & -.49 & -.49 \\
Hours & .72 & .56 & & \\
Dividends & .92 & .43 & &
\end{tabular}

\section{A Appendix}

This appendix describes a methodology for deriving the backward and forward weight distributions implied by error-correction equations of a PAC model.

\section{A.1 The Model}

The Euler equation for the loss function in (1) is, as shown in Tinsley (1993), ${ }^{13}$

$$
y_{t}^{*}=\left[1+\sum_{k=1}^{m} b_{k}\left[(1-L)(1-\beta F)^{k}\right]\right] y_{t} \text {, }
$$

which can be equivalently expressed as,

$$
\begin{aligned}
y_{t}^{*} & =\gamma A(\beta F) A(L) y_{t}, \\
& \equiv C(L, \beta F) y_{t}
\end{aligned}
$$

\footnotetext{
${ }^{13} L$ and $F$ are the conventional lag and lead operator, respectively.
} 
where $\gamma^{-1}=A(1) A(\beta)$ is a constant and ensures that $y_{t}=y_{t}^{*}$ in equilibrium. The lag polynomial, $A(z)$, is a function of the cost coefficients in (1), and, expanded, is given by,

$$
A(x)=a_{0}+a_{1} x+a_{2} x^{2}+\cdots+a_{m} x^{m} .
$$

The Euler condition implies the following PAC decision rule for for the $\mathrm{I}(1)$ decision variable, $y_{t}$,

$$
\begin{aligned}
\Delta y_{t} & =-A(1)\left(y_{t-1}-y_{t-1}^{*}\right)+A^{*}(L) \Delta y_{t-1}+A(\beta) A(1)\left\{\sum_{i=0}^{\infty} \iota_{m}^{\prime}[I-G]^{-1} G^{i} \iota_{m} \Delta y_{t+i}^{*}\right\} \\
& \equiv-A(1)\left(y_{t-1}-y_{t-1}^{*}\right)+A^{*}(L) \Delta y_{t-1}+S_{t}^{1}\left(G, \Delta y^{*}\right)
\end{aligned}
$$

where $A(L)=A(1) L+\left[1-A^{*}(L) L\right](1-L), G$ is an $m m \times m$ companion matrix of the lead polynomial, $A(\beta F),{ }^{14}$ and the $m$-dimensional vector $\iota_{m}=[0, \ldots, 0,1]^{\prime}$ selects elements of the bottom row of $G$. Given a consistent estimate of the coefficients $A(1)$ and $A^{*}(L)$ in (8), the PAC polynomial $A(L)$ is uniquely identified.

\section{A.2 Deriving Cost Parameters from the Lag Polynomial}

An important property is that $A(\beta F) A(L)$ is "self-reciprocal" with the property that the coefficient on each term $L^{j}$ is the same as the coefficient on $(\beta F)^{j}$. Expanding $C(L, \beta F)$ yields,

$$
c_{m} L^{m}+\cdots+c_{1} L+c_{0}+c_{1}(\beta F)+\cdots+c_{m}(\beta F)^{m}
$$

where

$$
c_{i}=\gamma \sum_{k=j}^{m} a_{m+j-k} a_{m-k} \beta^{m-k} .
$$

The cost coefficients, $b_{k}$ can be retrieved from the estimated lag polynomial coefficients

\footnotetext{
${ }^{14} G$ is the "bottom-row" companion matrix featuring the requisite zeros and ones in the top portion, and elements, $-a_{m} \beta^{m},-a_{m-1} \beta^{m-1}, \ldots,-a \beta$ in the bottom row, where the $a$ 's are the coefficients in $A(L)$.
} 
embedded in $A(L)$ by expanding $1+\sum_{k=1}^{m} b_{k}[(1-L)(1-\beta F)]^{k}$ and equating like terms in $L$ in this and (9).

\section{A.3 Mean Leads and Lags}

Letting $T(L, \beta F)=C(L, \beta F)^{-1}$, the mean lag and mean lead are defined by,

$$
\begin{array}{r}
\text { Mean Lag }=\frac{T_{L}(1,0)}{T(1,0)}=-\frac{A(0) A_{L}(1)}{A(1)}=-\frac{A_{L}(1)}{A(1)} \\
\text { Mean Lead }=\frac{T_{F}(0, \beta)}{T(0, \beta)}=-\frac{A_{F}(\beta) A(0)}{A(\beta)}=-\frac{A_{F}(\beta)}{A(\beta)},
\end{array}
$$

where $T_{i}$ is the derivative of $T(L, \beta F)$ with respect to $i=L$ or $F$. For the price equation, the mean lead and mean lag are the same: $3.9 .^{15}$

\section{A.4 The Lead and Lag Weights}

Equation (8) may be solved as follows. Write,

$$
A(L) y_{t}=A(\beta F)^{-1} A(\beta) A(1) y_{t}^{*} \equiv \bar{y}_{t}^{e}
$$

Define the vector $z_{t}=\left[z_{t}^{(m)}, z_{t}^{(m-1)}, \ldots, \ldots, y_{t}\right]^{\prime}$, where $z_{t}^{(m)}=F^{m} y_{t}$ is the $m$-th lead of $y_{t}$. Using the previously defined companion matrix, $G$, the right hand side of (13) can accordingly be rewritten as the difference equation

$$
z_{t}=G z_{t+1}+Y_{t}^{*}
$$

Equation (14) can be solved by forward recursion, where $Y_{t}^{*}=\left[0,0, \cdots, y_{t}^{*}\right]^{\prime}$. Repeated substitution of $y_{t}$ onto itself yields,

$$
z_{t}=A(\beta) A(1) \sum_{i=0}^{\infty} G^{i} Y_{t+i}^{*}
$$

\footnotetext{
${ }^{15}$ For $\beta=.98, A(L)=1-1.26 L+.08 L^{2}+.26 L^{3}, A(\beta F)=-1.23 L+.08 L^{2}+.24 L^{3}$.
} 


$$
\bar{y}_{t}^{e}=A(\beta) A(1) \iota_{m}^{\prime} \sum_{i=0}^{\infty} G^{i} \iota_{m} Y_{t+i}^{*}
$$

Combining equation (13) with (16), we have,

$$
A(L) y_{t}=\bar{y}_{t}^{e}
$$

which can be solved by backward recursion. Again, define an $m \times m$ companion matrix $H$ of the lag polynomial, $A(L)^{16}$. Using $H$ and the vector, $x_{t}=\left[x_{t}^{(m)}, x_{t}^{(m-1)}, \cdots, \ldots, y_{t}\right]^{\prime}$, where $x_{t}^{(m)}=L^{m} y_{t},(17)$ is cast into first order,

$$
x_{t}=H x_{t-1}+z_{t},
$$

which has the solution,

$$
\begin{aligned}
x_{t} & =\sum_{j=0}^{\infty} H^{j} z_{t-j} \\
y_{t} & =\iota_{m}^{\prime} \sum_{j=0}^{\infty} H^{j} \iota_{m} z_{t-j}, \\
& =A(\beta) A(1) \iota_{m}^{\prime} \sum_{j=0}^{\infty} H^{j} \iota_{m} \iota_{m}^{\prime} \sum_{i=0}^{\infty} G^{i} \iota_{m} Y_{t+i}^{*}, \\
& =A(\beta) A(1) \iota_{m}^{\prime} \sum_{j=0}^{\infty} \sum_{i=0}^{\infty} H^{j} \iota_{m} \iota_{m}^{\prime} G^{i} \iota_{m} Y_{t+i}^{*} .
\end{aligned}
$$

From this we may cull the weights associated with $y^{*}$ for all dates, $\{t-k, t-k+$ $1, \cdots, t-1, t, t+1, \cdots, t+k-1, t+k\}$, where $k$ is arbitrarily large. The above equation consists of the following sum,

$$
\begin{aligned}
y_{t} & =\iota_{m}^{\prime} H^{k} \kappa\left[G^{0} \iota_{m} Y_{t-k}^{*}+G^{1} \iota_{m} Y_{t-k+1}^{*}+\cdots+G^{k} \iota_{m} Y_{t}^{*}\right] \\
& +\iota_{m}^{\prime} H^{k-1} \kappa\left[G^{0} \iota Y_{t-k}^{*}+G^{1} \iota_{m} Y_{t-k+1}^{*}+\cdots+G^{k} \iota_{m} Y_{t+1}^{*}\right]
\end{aligned}
$$

\footnotetext{
${ }^{16} \mathrm{H}$ is the "bottom-row" companion matrix similar to $G$, featuring the requisite zeros and ones in the top portion, and elements, $-\alpha_{m},-\alpha_{m-1}, \ldots,-\alpha_{1}$ in the bottom row.
} 


$$
+\iota_{m}^{\prime} H^{0} \kappa\left[G^{0} \iota_{m} Y_{t}^{*}+G^{1} \iota_{m} Y_{t+1}^{*}+\cdots+G^{k} \iota_{m} Y_{t+k}^{*}\right]
$$

where $\kappa=A(\beta) A(1) \iota_{m} \iota_{m}^{\prime}$. For each date then, the distributed lead and lag weights are,

$$
\begin{aligned}
w_{t-k} & =\iota_{m}^{\prime} H^{k} \kappa G^{0} \iota_{m} \\
w_{t-s} & =\iota_{m}^{\prime} \sum_{i=0}^{k-s} H^{i+s} \kappa G^{i} \iota_{m} \\
& \cdot \\
& \cdot \\
w_{t} & =\iota_{m}^{\prime} \sum_{i=0}^{k} H^{i} \kappa G^{i} \iota_{m} \\
& \cdot \\
& \cdot \\
w_{t+s} & =\iota_{m}^{\prime} \sum_{i=0}^{k-s} H^{i} \kappa G^{i+s} \iota_{m} \\
w_{t+k} & =\iota_{m}^{\prime} H^{0} \kappa G^{k} \iota_{m},
\end{aligned}
$$

where $\sum_{s=-\infty}^{\infty} w_{t+s}=1$. 


\section{References}

Brayton, F., E. Mauskopf, D. Reifschneider, and J.C Williams, "The Role of Expectations in the FRB/US Macroeconomic Model," Federal Reserve Bulletin, 1997, 83, $227-245$.

Brayton, Flint and Peter A. Tinsley, "A Guide to FRB/US: A Macroeconomic Model of the United States," Finance and Economics Discussion Series 96-42, Federal Reserve Board, Washington, D.C. (eds.), 1996.

, Andrew Levin, Ralph Tryon, and John C. Williams, "The Evolution of Macro Models at the Federal Reserve Board," Carnegie-Rochester Conference Series on Public Policy, 1997, 43.

Calvo, Guillermo A., "Staggered Prices in a Utility-Maximizing Framework," Journal of Monetary Economics, 1983, 12, 383-398.

Eisner, R. and R. Strotz, Determinants of Business Investment, Impacts of Monetary Policy, CMC, Englewood Cliffs, N.J.: Prentice-Hall, 1963.

Kozicki, Sharon and Peter A. Tinsley, "Vector Rational Error Correction," Journal of Economic Dynamics and Control, 1999, 23, 1299-1327.

Tinsley, Peter A., "Fitting Both Data and Theories: Polynomial Adjustment Costs and Error-Correction Decision Rules,' Finance and Economics Discussion Series 93-21, Federal Reserve Board, Washington, D.C. 1993.

and Reva Krieger, "Asymmetric Adjustments of Price and Output," Economic Inquiry, 1997, 35, 631-632.

von zur Muehlen, Peter, "Dynamic Factor Demand with Final and Intermediate Inventories,” 1993. Unpublished Manuscript, Federal Reserve Board, Washington, D.C. 
Figure 1: Distributions of weights in spending equations

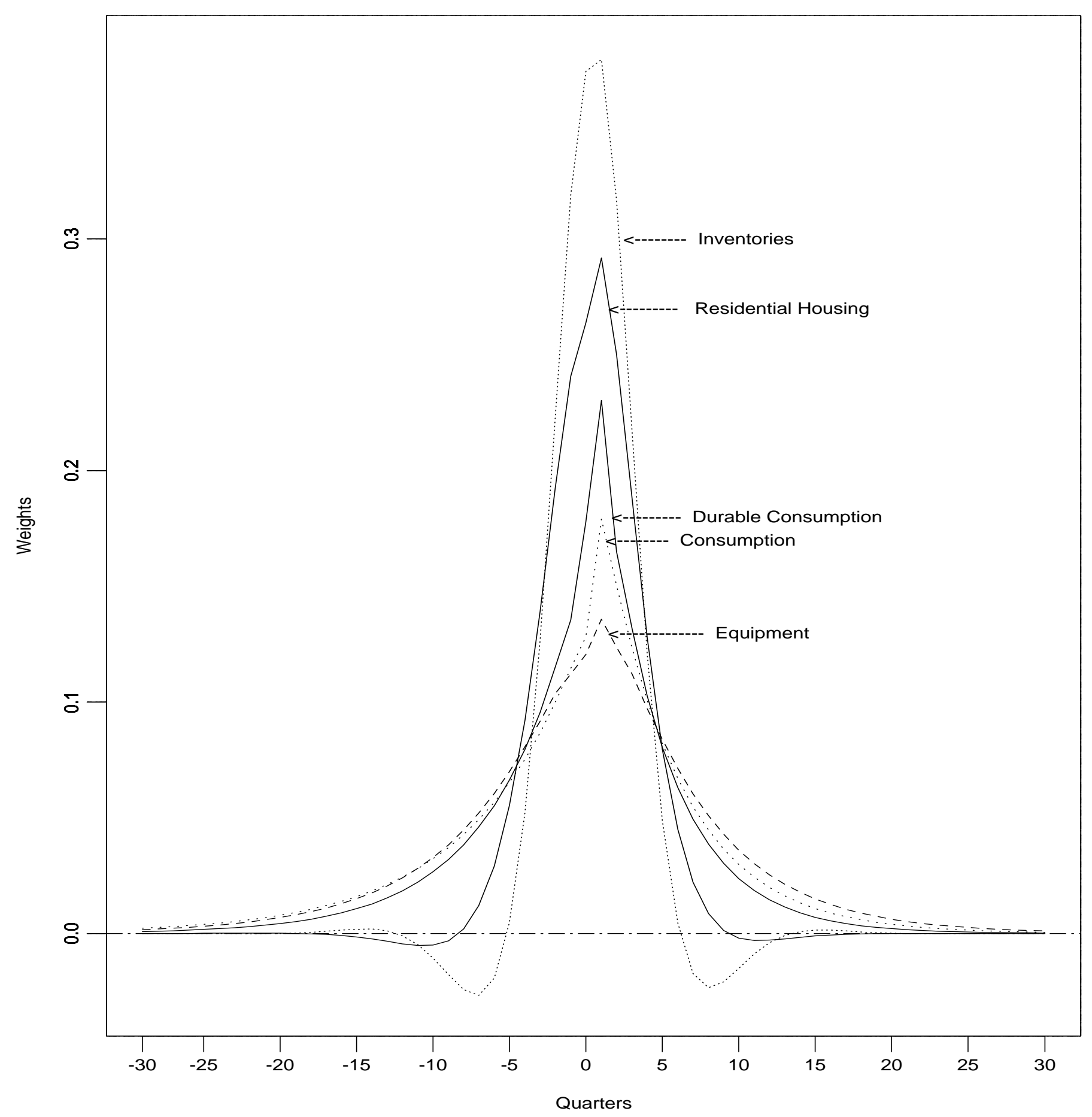


Figure 2: Distributions of weights in price and dividend equations

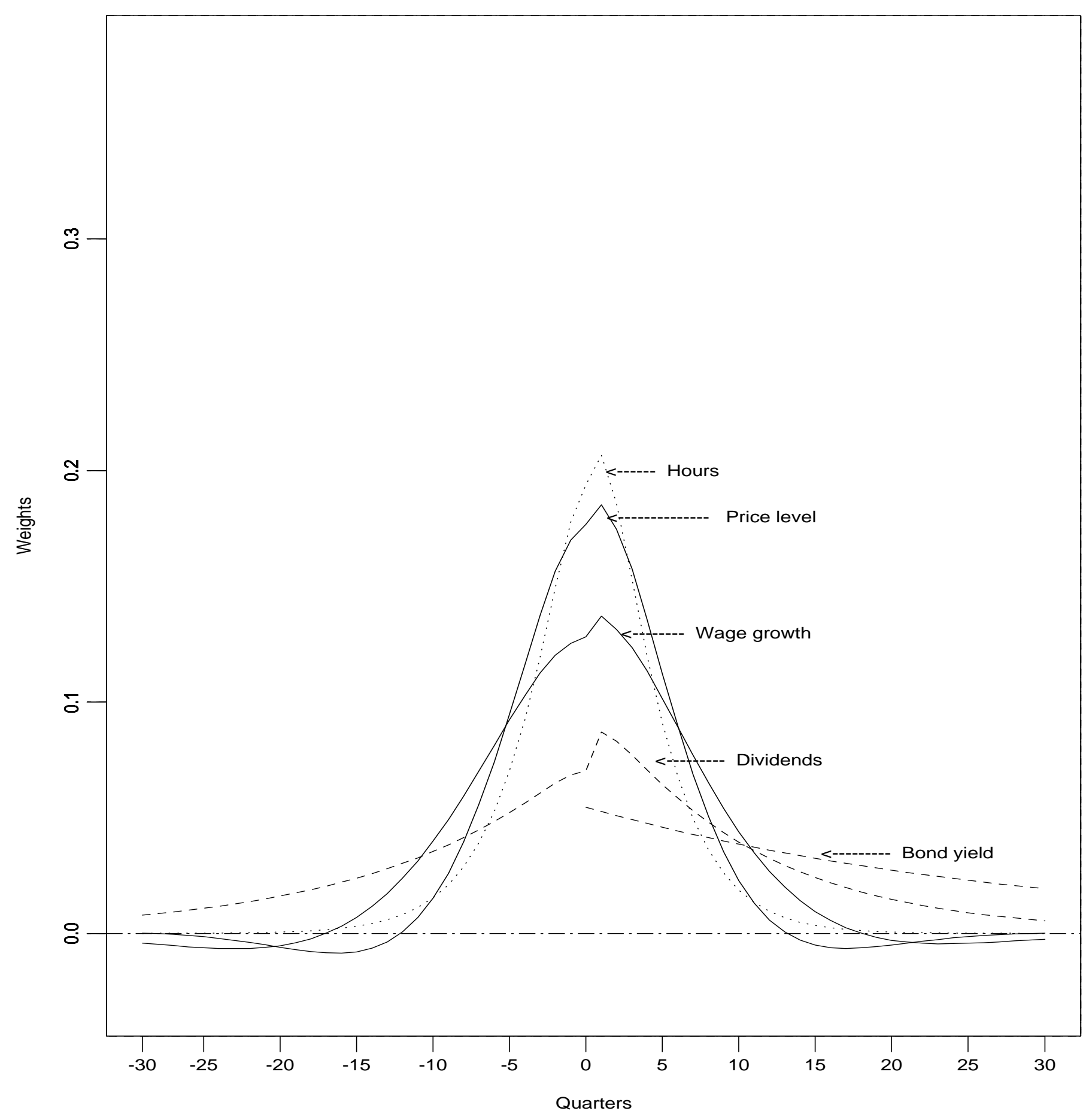

